\title{
LETTERS
}

\section{Buprenorphine-naloxone "microdosing": an alternative induction approach for the treatment of opioid use disorder in the wake of North America's increasingly potent illicit drug market}

After reviewing the guideline by Bruneau and colleagues, ${ }^{1}$ we agree that appropriate therapy should be used to manage opioid use disorders, with buprenorphine-naloxone being a first-line option. A substantial limitation of this medication, however, is the need for patients to abstain from opioid use for a period of time before starting buprenorphine-naloxone. This allows adequate time for the elimination of systemic full opioid agonists to avoid precipitation of opioid withdrawal with the use of buprenorphine-naloxone (a partial opioid agonist with high affinity for the $\mu$-opioid receptor). In the current fentanyl era, however, successfully achieving this "opioid washout" can be a challenge. Although fentanyl has a rapid onset and short duration of action, it is lipophilic, resulting in distribution to the peripheral tissues in a manner that is not dose dependent. ${ }^{2,3}$ Consequently, continuous and prolonged use of fentanyl can result in increased volume of distribution systemically with slow dissipation overall. ${ }^{2}$ Accordingly, the pharmacokinetics of fentanyl combined with its high prevalence in the illicit drug market appear (at least anecdotally) to be increasing the incidence of precipitated withdrawal during the induction process of buprenorphine-naloxone. This occurs despite patients objectively being in moderate to severe opioid withdrawal before starting the medication.
To address this, some prescribers in British Columbia (and likely beyond) are adopting a "microdosing" approach for buprenorphine-naloxone inductions. Officially recognized as the Bernese method, ${ }^{4}$ microdosing involves prescribing buprenorphine-naloxone in a small initial dose (e.g., $0.5 \mathrm{mg} / 0.125 \mathrm{mg}$ ) with incremental increases to both dose and frequency over time (Box 1). Coinciding with this, patients can continue to use other opioids (either prescribed or illicit) until a therapeutic dose of buprenorphine-naloxone has been achieved (e.g., usually $>8 \mathrm{mg}$ daily), when full opioid agonists are generally discontinued. ${ }^{4}$ The induction process takes place over a 7 - to 10 -day period and has been associated with substantial success. Patients have consistently reported that the induction process is well tolerated, with a reduction or elimination of cravings and avoidance of precipitated withdrawal. ${ }^{4,5}$

Although further research evaluating "microdosing" with buprenorphinenaloxone is certainly needed (we found only 2 case reports and 1 small case series), 4,5 adoption of this prescribing approach may offer a safe and effective alternative strategy for buprenorphine-

Box 1: Outpatient microdosing induction schedule for buprenorphine-naloxone

- Day 1: 0.5 mg once a day

- Day 2: $0.5 \mathrm{mg}$ twice a day

- Day 3: $1 \mathrm{mg}$ twice a day

- Day 4: 2 mg twice a day

- Day 5: 3 mg twice a day

- Day 6: 4 mg twice a day

- Day 7: $12 \mathrm{mg}$ (stop other opioids) naloxone induction among patients with an opioid use disorder. In the wake of an ever-changing illicit drug market, innovation (with simultaneous evaluation) is needed to help turn the tide on North America's opioid crisis.

\section{Privia A. Randhawa BScH MPH}

Medical student, Department of Medicine, University of British Columbia,

Vancouver, BC

\section{Rupinder Brar MD}

Family and addictions physician, British Columbia Centre on Substance Use; Department of Medicine, University of British Columbia, Vancouver, BC

\section{Seonaid Nolan MD}

Assistant professor, British Columbia

Centre on Substance Use; Department of Medicine, University of British Columbia, Vancouver, BC

Cite as: CMAJ 2020 January 20;192:E73. doi: $10.1503 / \mathrm{cmaj} .74018$

\section{References}

1. Bruneau J, Ahamad K, Goyer M-È, et al. Management of opioid use disorders: a national clinical practice guideline. CMAJ 2018;190:E247-57.

2. McClain DA, Hug CC Jr. Intravenous fentanyl kinetics. Clin Pharmacol Ther 1980;28:106-14.

3. Holley FO, van Steennis C. Postoperative analgesia with fentanyl: pharmacokinetics and pharmacodynamics of constant-rate i.v. and transdermal delivery. Br J Anaesth 1988;60:608-13.

4. Hämmig R, Kemter A, Strasser J, et al. Use of microdoses for induction of buprenorphine treatment with overlapping full opioid agonist use: the Bernese method. Subst Abuse Rehabil 2016;7:99-105.

5. Klaire S, Zivanovic R, Barbic SP, et al. Rapid micro-induction of buprenorphine/naloxone for opioid use disorder in an inpatient setting: case series. Am J Addict 2019;28:262-5.

Competing interests: None declared. 